\title{
Construindo um design para a economia verde
}

Shaping a design for the green economy 
Gabriela Garcez Duarte ${ }^{1}$

ORCID: https://orcid.org/0000-0002-0359-5615

\section{Aguinaldo dos Santos ${ }^{2}$}

ORCID: https://orcid.org/0000-0002-8645-6919

[resumo] 0 presente artigo procura levantar fundamentos para a construção de um design orientado pela economia verde, como um modo de se fomentar a transição para a sustentabilidade. 0 conceito de economia verde tem sido legitimado como uma economia na qual o crescimento está dissociado da exploração do meio ambiente, enquanto viabiliza a regeneração do mesmo, reduzindo os danos, aprimorando o bem-estar humano e a coesão social. Apesar de o crescimento econômico ilimitado provocar impactos negativos na própria economia e nas outras duas dimensões da sustentabilidade, a ambiental e a social, entende-se que uma expansão pautada na economia verde é relevante em contextos nos quais se necessita de maior empregabilidade e aumento da geração de renda, bem como de aprimoramento no manejo dos recursos naturais, entre outras questões relacionadas. Assim, os autores consideram que um design para a economia verde possa vir a propor soluções sistêmicas que contribuam na solução desse tipo de problema, de modo gradativo e a longo prazo. Logo, objetiva-se realizar uma revisão teórica que contemple essa proposta econômica, e os conceitos derivados dela, a fim de identificar abordagens e estratégias relativos ao design. Como resultado, o estudo contextualiza a legitimação da economia verde e investiga as prescrições de ordem produtiva das economias criativa, circular, compartilhada, colaborativa e distribuída e do comércio justo. Desse modo, identifica-se as estratégias de desmaterialização, manutenção e reparo, bem como de simbiose industrial e as abordagens de criação de sistema de produto mais serviço sustentável e de valorização intelectual e/ou cultural.

\section{[palavras-chave] Design. Economia verde. Desenvolvimento sustentável.}

\footnotetext{
1 Doutoranda pela Universidade Federal do Paraná (UFPR) e Mestre pelo Centro Universitário SENAC SP. E-mail: gabriela.garcezduarte@gmail.com. Lattes: http://lattes.cnpq.br/9478452525734470.

2 Doutor pela Universidade Federal do Paraná (UFPR). E-mail: a.santos@ufpr.br. Lattes: http://lattes. cnpq.br/7834194247765889.
} 
[abstract] This article seeks to raise foundations for the construction of a Design oriented by the green economy, as a way to foster the transition to sustainability. The concept of green economy has been legitimized as an economy in which growth is dissociated from the environment exploitation, while enabling its regeneration, reducing environmental risks, improving human welfare and social cohesion. Although unlimited economic growth generates negative impacts on the economy itself and on the other two dimensions of sustainability, the environmental and the social, it is understood that growth based on the green economy is relevant in contexts in which greater employability and increase in income generation are required, as well as improvement in the management of natural resources, among other related issues. Thus, the authors consider that a Design for the green economy may propose systemic solutions that contribute to the solution of this type of problem, gradually and in the long term. Therefore, it is aimed to carry out a theoretical review that contemplates this economic proposal and the concepts derived from it, in order to identify approaches and strategies related to Design. As a result, the study contextualises the legitimation of the green economy and investigates the productive order prescriptions of the creative, circular, shared, collaborative, distributed and fair trade economy. In this way, the strategies of dematerialisation, maintenance and repair, as well as industrial symbiosis and the approaches of creating sustainable product plus service system and of intellectual and/or cultural valorisation are identified.

[keywords] Design. Green economy. Sustainable development.

Recebido em: 07-01-2021

Aprovado em: 27-04-2021 


\section{Por que um design para a economia verde?}

O conceito de economia verde tem sido legitimado pela United Nations Environment Programme (Unep, 2019) como uma economia na qual o crescimento econômico possa ocorrer enquanto regenera o meio ambiente e reduz os danos, aprimorando o bem-estar humano e a coesão social. Apesar de esse conceito ser sendo disseminado desde o fim do século XX (PEARCE; MARKANDYA; BARBIER, 1989; PEARCE; MORAN, 1994), seu potencial de investigação e aplicação ainda possui amplo espaço de exploração no design.

Dar enfoque na economia verde é um modo de incentivar o desenvolvimento sustentável a partir do próprio sistema econômico predominante, o qual tem como base o capitalismo. Isto é colocado já que a economia verde não refuta o crescimento econômico, mas o adota como motivação para que nações e organizações empresariais busquem pela sustentabilidade ${ }^{3}$. Assim, os autores acreditam na eficiência ${ }^{4}$ da economia verde uma vez que ela se contrapõe ao crescimento econômico ilimitado e exponencial ${ }^{5}$, o qual tem sido estimulado por meio da aceleração industrial com base na exploração também ilimitada dos recursos naturais e do capital social. Isto tem ameaçado a sustentabilidade do planeta como um sistema interdependente (BRUNDTLAND, 1989; SANDRONI, 1999; ALTENBURG; RODRIK, 2017).

Observa-se, por exemplo, que apesar da contínua expansão econômica das nações desenvolvidas, o PIB per capita não aumentou. Mais da metade da população mundial vive com menos de US\$ 5,50 por dia e 4,5 bilhões de pessoas ainda vivem sem saneamento básico. Enquanto isso, os 2.153 bilionários mais ricos do mundo possuem mais riqueza do que 4,6 bilhões de pessoas, o que representa aproximadamente $60 \%$ da população. Seguindo a mesma lógica da desigualdade socioeconômica, as empresas multinacionais concentram em torno de $40 \%$ de seus ganhos nos chamados paraísos fiscais. E as taxas globais de impostos sobre as mesmas - que poderiam compensar esse desequilíbrio caíram de 40,38\% para 24,18\% de 1980 a 2019 (MARKANDYA; BARBIER, 2014; ALTENBURG; RODRIK, 2017; SUSTAINABLE DEVELOPMENT, 2019; ONU, 2020; OXFAM, 2020).

\footnotetext{
3 Adota-se o termo sustentabilidade como o equilíbrio das dimensões econômica, ambiental e social (Manzini; Vezzoli, 2002). Todavia, não existe um único entendimento de como adotá-la, já que cada contexto é único e dinâmico (GEELS, 2010). Assim, as soluções aqui propostas não são absolutas, são pautadas no tempo humano e de acordo com o contexto do problema elencado.

${ }^{4}$ Usa-se o termo eficiência para designar a condição de desempenho de uma solução com menos falhas possíveis ao longo do desenvolvimento das soluções propostas.

Para abordar outro assunto (o do crescimento populacional), Meadows et al. explicam que "uma quantidade exibe crescimento exponencial quando esta cresce em uma porcentagem constante do todo em um período de tempo constante" (MEADOWS et al., 1972, p. 27). Para simplificar, os autores expõem que seria útil pensar no crescimento exponencial em termos de dobrando no tempo. Um exemplo fornecido por eles é o de uma colônia de levedura na qual cada célula divide-se em duas a cada 10 minutos e, portanto, para cada única célula, depois de 10 minutos, haverá duas células e um aumento de 100\%; logo, após os próximos 10 minutos, haverá quatro células, depois oito e, depois, 16 e assim por diante (MEADOWS et al., 1972). Harvey (2016) cita que o crescimento econômico ilimitado tem seguido, desde os anos de 1970, essa mesma lógica exponencial.
} 
Esses dados ressaltam que as atividades econômicas têm sido mensuradas pelos interesses localizados de empresas e nações, sem considerar as externalidades ${ }^{6}$. Assim, corroboram com a escassez de recursos naturais e inadequações sociais que ameaçam a própria sustentação do sistema econômico (PEARCE; MARKANDYA; BARBIER, 1989; PEARCE; MORAN, 1994; LOISEAU et al., 2016).

Algumas consequências disso podem ser claramente observadas também no meio ambiente. Nos anos 2000, 60\% do ecossistema já havia sido utilizado de modo que desde então não se permite sua sustentabilidade. Os impactos mais preocupantes estão relacionados ao clima e às emissões globais de $\mathrm{CO} 2$ fóssil e concentração de gases de efeito estufa (GEE), resultantes essencialmente da atividade industrial. Alguns dados destacam, nesse panorama, o aumento da média da temperatura global, a qual, ao ser analisada entre 2015 e 2019, é considerada a mais quente desde a era pré-industrial (1850-1900). 0 aumento do nível do mar também tem se acelerado e a sua acidez cresceu $26 \%$ desde o começo da industrialização ${ }^{7}$. Já as emissões de CO2 que escalavam desde 2015 a 1\% anualmente, atingiu $2 \%$ em 2018, enquanto a quantidade de extração de matérias-primas global dobrou em 30 anos $^{8}$ (ALTENBURG; RODRIK, 2018; UNITED IN SCIENCE, 2019).

Logo, um sistema econômico que permita a regeneração do meio ambiente e a equidade social se faz necessário. No entanto, enquanto Schumacher (1973) e a corrente da economia ecológica propõem o decrescimento econômico, os autores entendem que este ainda é relevante em lugares e situações nos quais o aumento da empregabilidade e da geração de renda propiciam melhorias na saúde e na educação, reforçando positivamente a equidade social. 0 mesmo vale para o âmbito ambiental, pois, uma vez supridas as necessidades econômicas para o bem-estar dos indivíduos, o manejo dos recursos naturais tende a ser mais sustentável (BRUNDTLAND, 1989; ALTENBURG; RODRIK, 2017).

Assim, os autores consideram que um design para a economia verde possa vir a propor soluções pautadas na atividade criativa e industrial de modo adequado à busca pelo crescimento econômico - a qual é inerente ao sistema predominante e de difícil encerramento em curto período -, fomentando uma transição para a sustentabilidade ${ }^{9}$ de modo gradativo e a longo prazo.

\footnotetext{
${ }^{6}$ As externalidades são efeitos de implementações de ordem econômica. No caso da economia verde, as externalidades referem-se aos impactos ambientais e sociais, os quais devem ser previstos de modo que tragam benefícios à natureza e ao meio social (PEARCE; MARKANDYA; BARBIER, 1989; SANDRONI, 1999; PEARCE; MORAN, 1994).

7 Segundo o relatório, os níveis atuais de $\mathrm{CO} 2, \mathrm{CH} 4$ e N20 representam um aumento respectivo de 146\%, $257 \%$ e 122\% dos níveis da era pré-industrial (pré-1750) (UNITED IN SCIENCE, 2019).

8 Os dados apontam que foi de aproximadamente 36 bilhões de toneladas em 1980 para 85 bilhões de toneladas em 2013, representando um crescimento geral de 132\% (RODRIK; RODRIK, 2018).

9 Entende-se que as transições para a sustentabilidade ocorrem quando uma grande mudança tecnológica e/ou social atinge diferentes camadas da sociedade e transforma um paradigma. Assim, mudam-se regras científicas e de mercado, padrões culturais como o consumo, organizações e instituições (GEELS; SCHOT, 2007; GEELS, 2010). Neste artigo, portanto, entende-se que a dimensão econômica é fundamental para que uma transição para a sustentabilidade ocorra e, por isso, propõe a economia verde.
} 
Visando, portanto, construir um design pautado no conceito original de economia verde, este artigo tem como objetivo realizar uma revisão teórica que contemple essa proposta econômica, e os conceitos derivados dela, a fim de identificar quais abordagens e estratégias fundamentam essa elaboração. Com esse escopo, investiga-se a economia criativa, a circular, a compartilhada, a colaborativa, a distribuída e o comércio justo ${ }^{10}$. Desse modo, almeja-se que este estudo seja utilizado por profissionais e pesquisadores de design e de áreas afins como a moda, no entendimento e no desenvolvimento de modelos de negócios, sistemas, produtos e/ou serviços que buscam por alternativas economicamente prósperas e simultaneamente sustentáveis.

\section{Identificando conceitos}

0 tema geral deste artigo trata essencialmente das áreas de pesquisa do Design, da Sustentabilidade e da Economia. Dada a amplitude delas, optou-se por selecionar o material para a revisão teórica a partir de uma revisão bibliográfica sistemática (RBS). Com isso, foi possível filtrar os conceitos econômicos que mais têm sido utilizados no Design para a Sustentabilidade (DpS) ${ }^{11}$ e derivam da proposta da economia verde.

A RBS foi conduzida pela pergunta "Identifica os principais conceitos, abordagens e/ ou estratégias para a economia verde?". Para tanto, a pesquisa foi realizada a partir da fonte de dados do portal de periódicos da Capes (CAFE CAPES, 2019).

O levantamento dos conceitos econômicos que serão revisados foi identificado por meio da pesquisa realizada pelo conjunto de termos (string) "sustainability and economy", dada em um primeiro ciclo. Assim, com enfoque na sustentabilidade e na economia, foi possível elencar investigações recentes (de até 10 anos) e de palavras-chave relevantes a este estudo. A partir dessa busca, foi possível identificar os termos utilizados que tratam de sustentabilidade e economia e, então, foram verificadas as incidências de estudos dentro do design em um segundo ciclo, conforme resultado especificado abaixo (tabela 1), para posteriormente selecionar o material da revisão teórica.

\footnotetext{
10 Esse levantamento de conceitos vem da Revisão Bibliográfica Sistemática a qual será explicada posteriormente neste artigo.

${ }^{11}$ Considera-se o DpS uma corrente de estudos e aplicações que propõe que o design seja sustentado nas três dimensões da sustentabilidade, a ambiental, a social e a econômica (MANZINI; VEZZOLI, 2002; VEZZOLI et al., 2018).
} 
TABELA 1 - TERMOS LEVANTADOS E VOLUME DE ARTIGOS SELECIONADOS

\begin{tabular}{|c|c|c|}
\hline $1^{\circ}$ CICLO / STRINGS & TOTAL LEVANTADOS & TOTAL SELECIONADOS \\
\hline Sustainability and economy & 440 & 79 \\
\hline \multicolumn{3}{|c|}{$2^{\circ}$ CICLO / DESIGN “and": PALAVRAS-CHAVE LEVANTADAS } \\
\hline Design and solidarity economy & 0 & 0 \\
\hline Design and ecological economy & 1 & 1 \\
\hline Design and fair trade & 4 & 1 \\
\hline Design and distributed economy & 4 & 2 \\
\hline Design and collaborative economy & 5 & 5 \\
\hline Design and sharing economy & 14 & 7 \\
\hline Design and circular economy & 16 & 10 \\
\hline Design and green economy & 17 & 9 \\
\hline Design and creativity economy & 21 & 9 \\
\hline TOTAL & & 123 \\
\hline
\end{tabular}

FONTE: DUARTE, Gabriela Garcez (2020). Design para a economia verde: aprendizagem reflexiva em organizações. No prelo. Imagem obtida mediante cópia digital do próprio documento.

A revisão teórica apresentada a seguir é resultado de uma seleção posterior aos 123 artigos escolhidos. Isto se deu com base na verificação dos resumos. As referências que permitiam a identificação de conceitos, abordagens e/ou estratégias para a economia verde foram consultadas. Algumas referências a mais, citadas nos materiais levantados, também foram utilizadas. Logo, os conceitos vêm das referências que permitiram a compreensão tanto da ideia de economia verde quanto das noções de economia criativa, economia circular, economia compartilhada, economia colaborativa, economia distribuída e comércio justo ${ }^{12}$.

\footnotetext{
12 Os conceitos encontrados, além de economia verde e os artigos selecionados para a leitura, são aqui enumerados com suas fontes principais, a partir da ordem de maior incidência para a de menor: (i) economia criativa (CASTRO e FIGUEIREDO, 2016; DANTAS e TARALLI, 2017; SANTOS et al., 2019); (ii) economia circular (BRAUNGART et al., 2007; BAKKER et al., 2014; LOISEAU et al., 2016; SCHEEL, 2016; DEN HOLLANDER et al., 2017; DESPEISSE et al., 2017; MENDOZA et al., 2017; ELLEN MACARTHUR FOUNDATION, 2020); (iii) economia compartilhada (KULP e KOOL, 2015; LIGHT e MISKELLY, 2015; VERBOVEN e VANHERCK, 2016; ESLER, 2017; PETROPOULOS, 2017; VASQUES et al., 2017; GAN et al., 2018; VEZZOLI et al., 2019); (iv) economia colaborativa (BARNES e MATTSSON, 2016; DE RIVERA et al., 2017); (v) economia distribuída (ULIANA e SANTOS, 2017; SANTOS et al., 2019; VEZZOLI et al., 2020); e (vi) comércio justo (BARBIER e MARKANDYA, 2013; AMBEC, 2014; KHMARA e KRONENBERG, 2018). Os artigos identificados a partir do string design and green economy foram analisados por meio de seus títulos e resumos e descartados aqueles cujo termo design foi utilizado para significar criação/desenvolvimento sem estar relacionado à área de design. Aqueles que permaneceram não tratavam diretamente do conceito, mas dos acima descritos.
} 


\section{A legitimação da economia verde e as oportunidades em design}

Introduzida por Pearce, Markandya e Barbier (1989), a proposta da economia verde serviu de alerta aos custos ambientais do crescimento econômico do Reino Unido em seu contexto de país desenvolvido. Nesse estudo, a degradação ambiental chegou a ser vinculada ao fato de o capital natural - o meio ambiente - "ser tratado como um recurso de custo zero quando, de fato, ele serve funções econômicas que possuem valor positivo" (PEARCE; MARKANDYA; BARBIER, 1989, p. 7). Segundo a perspectiva da economia verde, os produtos e os serviços deveriam tentar ao máximo empregar o valor econômico que o ambiente natural provê na tentativa de superar essa falha governamental e mercadológica.

A lacuna dos mercados estaria na falta de captação do valor da biodiversidade e dos benefícios da conservação ambiental local e global. Já a dos governos viria do modo como eles intervêm no funcionamento de setores do mercado que exploram os recursos naturais. Nesse sentido, Pearce e Moran (1994) demonstraram, por exemplo, que os subsídios para atividades de diferentes esferas (local, nacional ou global) tendem a ir contra a conservação ambiental ao favorecer a exploração do meio ambiente sem contabilizar a perda da biodiversidade ${ }^{13}$.

Os estudos originários da economia verde também questionaram a relação entre riqueza material e felicidade humana. Pearce, Markandya e Barbier (1989) contestaram se uma nação que já rica deve continuar buscando crescimento econômico. A falta de igualdade social, os baixos níveis de confiança nos outros e nas instituições, o aumento de doenças mentais, o abuso de drogas e álcool e os rompimentos familiares eram aspectos negativos em destaque na Grã Bretanha, os quais influenciaram esse questionamento (MARKANDYA; BARBIER, 2014; ILO, 2011, 2018).

Foi a partir de protocolos e acordos internacionais, como o Protocolo de Quioto (KYOTO PROTOCOL, 1998), o Global Green New Deal (GGND, 2009), a Conferência para o Clima (ONU, 2009) e a Green Economy Inclusiva (ONU, 2012), que se impulsionou o levantamento de recursos financeiros, políticas e outros instrumentos para a aplicação da economia verde ${ }^{14}$.

No âmbito do design, no entanto, observa-se uma lacuna de prescrições que, inicialmente, foi preenchida na RIO+20 (2012). Assim, a partir da perspectiva dos protocolos e acordos elaborados sob o panorama macroeconômico acima mencionado, foram identificadas métricas na tentativa de suprir o setor produtivo. Com isso, foram propostas as seguintes estratégias em eco-design:

\footnotetext{
13 Um exemplo dado por Pearce e Moran (1994) é o desmatamento para a prática da pecuária no Brasil e a irrigação para a agricultura no Paquistão e na Califórnia.

${ }^{14}$ Alguns dos aspectos mais relevantes para o investimento na bolsa de valores a respeito de ações chamadas verde são títulos de empresas que seguem as diretrizes resultantes de protocolos e acordos, como o GGND (2009), o GEI (2012) e os ODS (ONU, 2015) (PHALEN, 2020).
} 
QUADRO 1 - ESTRATÉGIAS PARA UMA AVALIAÇÃO DE CICLO DE VIDA VOLTADA À ECONOMIA VERDE

1. Redesenhar produtos e/ou modelos comerciais para que a mesma funcionalidade possa ser entregue com fundamentalmente menos uso de materiais e energia.

2. Reciclar resíduos de processos internos, incluindo águas, calor de alta temperatura, contrapressão etc.

3. Introduzir novas tecnologias (mais limpas) e melhorar a eficiência dos processos existentes para dar um salto e estabelecer novos modos de produção que, fundamentalmente, sejam mais eficientes em relação a material e à energia.

4. Redesenhar sistemas, especialmente o de transporte, e a infraestrutura urbana a jusante, para utilizar insumos menos intensivos em recursos.

5. Investir na eficiência de materiais, energia e água, assim como na transição para empregos verdes.

FONTE: Extraído de Unep e Setac (2012).

A partir dessas medidas, as decisões empresariais poderiam então se tornar mais efetivas para a economia verde (UNEP; SETAC, 2012). Contudo, observa-se a ênfase em soluções insulares, ou seja, mais concentradas em fases do ciclo de vida de produtos e serviços, no uso de matérias-primas de menor impacto ambiental e maior eficiência, bem como em energia limpa. Dessa maneira, as externalidades econômicas são deixadas um tanto quanto limitadas, já que não abordam questões sociais, a não ser a preocupação com o suprimento de mão de obra.

Com a organização dos Objetivos para o Desenvolvimento Sustentável (ODS), em 2015, pela Organização das Nações Unidas, a economia verde foi legitimada como uma perspectiva que propõe não apenas a preservação e a regeneração ambiental, mas também a responsabilidade de criar equidade social de indivíduos, grupos e nações. Dos 17 ODS e suas 169 metas voltadas para o ano de 2030, seis estão diretamente relacionados à economia verde: ODS 1 - Acabar com a pobreza em todas as suas formas, em todos os lugares; ODS 8 - Promover o crescimento econômico sustentado, inclusivo e sustentável, emprego pleno e produtivo e trabalho decente para todos; ODS 9 - Construir infraestruturas resilientes, promover a industrialização inclusiva e sustentável e fomentar a inovação; ODS 10 - Reduzir a desigualdade dentro dos países e entre eles; ODS 11 - Tornar as cidades e os assentamentos humanos inclusivos, seguros, resilientes e sustentáveis; e o ODS 12 - Assegurar padrões de produção e de consumo sustentáveis (ONU, 2015).

Sob a perspectiva do design, essa maior abrangência da economia verde para além do escopo do eco-design expõe uma oportunidade em se aproveitar as competências do DpS em abordagens sistêmicas. Dessa maneira, tendem a suprir problemas complexos e a viabilizar soluções que envolvam questões tanto tecnológicas quanto sociais (COSTA; DIEHL; SECOMANDI, 2019).

Em outubro de 2020, o GGND (2009) foi abordado pelos países integrantes da União Europeia de modo que possa ser integrado ao design a partir dos princípios da Bauhaus. Desse modo, nota-se que a chamada nova Bauhaus é uma iniciativa criativa e interdisciplinar que pretende inserir a perspectiva da economia verde, já que o GGND (2009) propõe justa- 
mente incentivos financeiros que proporcionem a transição para essa economia. A proposta europeia visa promover espaços mais econômicos e acessíveis, mobilizar os estudantes e profissionais criativos para reinventar um estilo de vida sustentável, aprimorar experiências com valores, como a funcionalidade, a simplicidade e a circularidade, e fornecer apoio financeiro às inovações (NEW EUROPEAN BAUHAUS, 2021).

Enquanto um design orientado para a economia verde é construído ainda com diversas lacunas, os conceitos derivados da mesma são mais utilizados nessa área criativa. Simultaneamente, as estratégias que eles englobam demonstram estar mais em acordo com abordagens sistêmicas, sem necessariamente descartar as soluções em eco-design consideradas aqui insulares. Logo, a fim de compreender de modo mais aprofundado as contribuições desses conceitos no DpS e possibilitar a construção de um design orientado para a economia verde, serão exploradas as suas prescrições no âmbito produtivo.

\section{Economia criativa}

O conceito de economia criativa abrange modelos de negócios que, por meio de valor simbólico, fomentem a geração de trabalho e renda. Tal valor é comumente originado por meio de ações criativas individuais e/ou coletivas que destacam o conteúdo intelectual e cultural. Assim, ao tratar essencialmente de recursos intangíveis, a economia criativa inclui diversos setores da indústria cultural. No Brasil, o Serviço Brasileiro de Apoio às Micro e Pequenas Empresas (SEBRAE) organiza esse tipo de economia em áreas estratégicas como consumo (design, arquitetura, moda e publicidade); mídias (editorial e audiovisual); cultura (patrimônio e artes, música, artes cênicas e expressões culturais); e tecnologia (P\&D, biotecnologia e TIC). Embora o design esteja alocado no âmbito do consumo, a amplitude das suas atividades permite que designers atuem nas outras áreas acima relacionadas. As soluções em economia criativa possuem características que vinculam inovação, sustentabilidade, diversidade cultural e/ou inclusão social (CASTRO; FIGUEIREDO, 2016; SANTOS et al., 2019; SEBRAE, 2020).

Esses aspectos costumam ser mensurados de acordo com a contrapartida oferecida à economia local. De um modo geral, os resultados esperados para a economia são: (i) o desenvolvimento setorial das atividades que compõem a economia criativa; (ii) o desenvolvimento territorial por meio da valorização das redes, dos costumes e da tradição local; (iii) o desenvolvimento transversal, de forma a criar valor e estimular a competitividade das empresas (CASTRO; FIGUEIREDO, 2016, p. 4, 114).

As inovações da economia criativa costumam incluir melhorias e/ou redesign de produtos. Nesse sentido, aspectos sociais são comumente evidenciados nas formas e/ou na escolha dos materiais. A inovação dos materiais pode ocorrer por transferência tecnológica de um segmento produtivo para outro ou por meio do redesenho de materiais tradicionais, com novos tipos de uso e/ou modo de produção. Observa-se ainda o investimento na comunicação com os diferentes segmentos de clientes, na busca por evidenciar as novas possibilidades funcionais dos materiais já existentes. Vale ressaltar que tais funções dos materiais, ao serem realocadas para finalidades práticas, estéticas e/ou simbólicas, destacam inovação no nível funcional (DANTAS; TARALLI, 2017). 
Ao procurar abranger soluções de contrapartida social e estrategicamente coordenadas em redes, as inovações da economia criativa podem alcançar o nível sistêmico. Nesse caso, são comuns propostas de valor vinculadas aos saberes, às tradições e/ou às técnicas produtivas de comunidades locais. Outras estratégias dentro da economia criativa promovem a inclusão social, a valorização do patrimônio cultural e ainda a revitalização e a valorização de áreas urbanas ou rurais em declínio. Logo, possibilitam resultados econômicos de longo prazo enquanto promovem bem-estar e coesão social (DANTAS; TARALLI, 2017; SANTOS et al., 2019).

\section{Economia circular}

Esse conceito propõe uma economia que valoriza a reintegração dos resíduos na cadeia de valor dos negócios por meio do fechamento do $l o o p^{15}$ do ciclo de vida (DE LOS RIOS; CHARNLEY, 2017). A fundação Ellen MacArthur (2020) aponta três princípios relativos à economia circular: (i) desenvolver soluções que eliminem os resíduos e a poluição; (ii) manter os produtos e materiais em uso; e (iii) regenerar os sistemas naturais. Desse modo, concentrada nos princípios I e II, grande parte das abordagens do design para a economia circular foca na recuperação dos artigos ou dos componentes por meio de manutenção, reparo, remodelação e remanufatura (reciclagem) (DESPEISSE et al., 2017). 0 incentivo do consumo do tipo faça você mesmo (do it yourself - D.I.Y.), que promove a manutenção dos itens e provoca empatia no consumidor, também faz parte das propostas de prolongamento do uso. Com essas estratégias, espera-se que o retorno financeiro mude da dependência de um ciclo pautado na aquisição, no uso e no descarte para a oportunidade de se criar valor a partir do que é comumente considerado um resíduo (BAKKER et al., 2014).

Dentro da proposta da economia circular, encontram-se outras bases teóricas. Uma delas é a ecologia industrial, também chamada de cluster ecológico. Ela prescreve uma espécie de simbiose entre as empresas localizadas próximas umas das outras. Assim, viabilizam a divisão de resíduos, subprodutos e refugos. Esse fluxo circular é uma proposta de crescimento regional desenhado para criar uma economia regenerativa que articule sistemas circulares capazes de alcançar benefícios sociais e ambientais para a população local. Nesse contexto, a ecoeficiência é uma estratégia que tem como objetivo economizar recursos financeiros e otimizar os insumos de produção ao máximo enquanto reduz a geração de resíduos e/ou os reutiliza (LOISEAU et al., 2016; SCHEEL, 2016; ELLEN MACARTHUR FOUNDATION, 2020).

A economia circular aborda pesquisa e desenvolvimento no âmbito de inovações relativas a melhorias e/ou ao redesign de produtos. Nesses casos, com foco no princípio III (regeneração dos sistemas naturais), usa-se a estratégia da ecoeficácia, a qual propõe a transformação dos produtos a partir dos seus materiais. Assim, possibilita uma relação orgânica de suporte aos sistemas ecológicos e ao crescimento econômico (BRAUNGART et al., 2007). A ecoeficácia "celebra a aplicação criativa e extravagante de materiais e permite um

\footnotetext{
50 termo loop tem sido utilizado no contexto produtivo, mesmo em português, para mencionar que o fim do ciclo de vida de um produto não é o seu descarte e sim sua reintrodução ao ciclo, como em um movimento circular (loop) sem fim.
} 
ciclo de vida curto do produto sob a condição de que todos os materiais retêm o status de recursos produtivos" (BRAUNGART et al., 2007, p. 2). Essa analogia com os organismos vivos e autogerenciáveis vão ao encontro de outra base teórica da economia circular, a da biomimética (ELLEN MACARTHUR FOUNDATION, 2020). Ceschin e Gasiuluzoy (2016) indicam que a biomimética é um modelo para a criação de formas que também podem contribuir na reintegração dos resíduos e ao estabelecimento de ciclos fechados.

\section{Economia compartilhada}

Esse conceito valoriza a utilização intensificada dos produtos e a otimização do uso pelo compartilhamento de itens, geralmente por meio de serviços, contribuindo para o que Mazini e Vezzoli (2002) chamam de desmaterialização ${ }^{16}$. Embora a troca seja uma prática fundamental da economia que precede o sistema monetário (LIGHT; MISKELLY, 2015), é o desenvolvimento e o acesso a tecnologias e à conexão de internet que permitem que esse tipo de economia seja difundida (KULP; KOOL, 2015; LIGHT; MISKELLY, 2015; GAN et al., 2018). Grande parte das inovações baseadas no conceito de economia compartilhada concentram-se sob o formato de plataformas on-line. Contudo, existem serviços voltados ao compartilhamento constituídos como plataformas físicas (VEZZOLI et al., 2019). Encontram-se ainda modelos híbridos de plataformas pautadas em informação on-line e pontos de contato físicos. Em todos, os proprietários de bens visam encontrar interessados no uso desses serviços e vice-versa, ocorrendo de modo gratuito ou pago.

Nessa dinâmica, Petropoulos (2017) destaca a participação de três elementos: (i) provedores que disponibilizam bens, recursos, tempo e/ou habilidades, bases de uso aos pares e mesmo serviço profissional; (ii) usuários que consomem os bens e os serviços oferecidos; e (iii) intermediários que conectam os provedores aos usuários e facilitam o pagamento. Sob o aspecto econômico, as plataformas de compartilhamento auxiliam indivíduos e empresas a maximizar os ganhos provenientes da posse de um produto ou da oferta de um serviço ao ampliar o acesso/uso por valores abaixo dos praticados no mercado (VERBOVEN; VANHERCK, 2016; GAN et al., 2018). Simultaneamente, possibilitam a participação econômica de atores, antes desfavorecidos competitivamente em relação às empresas já estabelecidas (PETROPOULOS, 2017). Os usuários abrem mão da posse fazendo apenas uso do produto e/ou da função.

A economia compartilhada, geralmente, impulsiona novas práticas, tendendo, assim, a provocar mudanças até mesmo no mercado. Algumas inovações relativas à economia compartilhada incitam os governos à criação de regulamentações de mercado, já que os modelos oportunizam tipos de trabalho com baixa formalização ${ }^{17}$.

Sistemas pautados na combinação híbrida de produto e serviço sustentáveis - chamados na comunidade do DpS de sistemas de produto mais serviço sustentáveis (S.PSS) -,

\footnotetext{
16 A desmaterialização é utilizada por Mazini e Vezzoli (2002) no sentido de que esse tipo de solução permite a diminuição da quantidade de produtos e/ou de matéria-prima utilizados.

17 Sabe-se que esse aspecto não é necessariamente um benefício social. Esse paradoxo será tratado nas considerações finais.
} 
quando voltados ao compartilhamento de bens e serviços, podem fomentar a geração de renda de atores sociais. Assim, valorizam a economia local (LIGHT; MISKELLY, 2015; GAN et al., 2018). Por não se pautar na produção em massa de novos produtos, pode diminuir consideravelmente os impactos ambientais da indústria de manufatura enquanto promove o crescimento econômico (VEZZOLI, 2020).

\section{Economia colaborativa}

As semelhanças entre o conceito de economia colaborativa e o de economia compartilhada são diversos e suas diferenças, complementares. Observa-se o destaque no uso de plataformas (digitais, físicas e híbridas) tanto na economia colaborativa quanto na compartilhada, buscando facilitar o acesso a bens e serviços. Assim, o papel de um stakeholder ${ }^{18}$ faz- $^{-}$ -se igualmente importante nesse conceito. Nota-se ainda que a otimização e a intensificação do uso são também valorizadas nas economias que visam a colaboração, porém em menor grau que na compartilhada (PETROPOULOS, 2017).

Porém, observa-se que o aspecto coletivo se sobrepõe ao individual na economia colaborativa a fim de resultar de fato em colaboração. Ou seja, mesmo que a ação seja realizada individualmente, ao acessar plataformas de colaboração, o sujeito deliberativamente colabora para a ação coletiva. Logo, entre os três papéis ativos nas plataformas - provedor, intermediário e usuário - observa-se o papel do consumidor provedor em destaque.

Seguindo a estratégia da economia colaborativa, os modelos de negócios voltam-se ao provimento de recursos tangíveis, como bens e recursos infraestruturais e intangíveis, como conhecimento e tempo. Nota-se que a colaboração se destaca em plataformas para: (i) financiamento coletivo, viabilizando economicamente diversos tipos de projetos individuais e/ou empresariais com ou sem fins lucrativos; (ii) compras coletivas, propiciando economicamente o acesso a bens e serviços por meio da redução dos preços em contraponto ao aumento do volume adquirido; e (iii) open source/open design, possibilitando o acesso ao conhecimento confiável dos pares e de forma gratuita e facilitando a cocriação por ferramentas de personalização e/ou customização enquanto diminui os riscos de estocagem dos negócios. Essas colaborações permitem o empoderamento do consumidor por meio do engajamento de pares e com ações de cunho empreendedor (RIVERA et al., 2017).

\section{Comércio justo}

Com foco original na valorização das atividades agrícolas e pecuárias, o comércio justo (fairtrade) aumenta o interesse de empresas e consumidores pela origem e pelos modos de cultivo de alimentos e matérias-primas naturais (BARBIER ; MARKANDYA, 2013). 0 comércio justo visa sobretudo estabelecer a negociação direta entre o produtor e o comprador, eliminando o excesso de burocracia e os custos decorrentes da presença de atravessadores,

\footnotetext{
${ }^{18} \mathrm{Em}$ português este termo pode ser traduzido como "parte interessada", porém em áreas relacionadas à Gestão de Negócios e ao Design observa-se o uso da palavra em inglês - stakeholder - para mencionar fornecedores e consumidores diretos e indiretos, sejam pessoas físicas ou jurídicas, desde que sejam possíveis participantes dos sistemas que compõe modelos de negócios e cadeias produtivas.
} 
além de instabilidades do mercado global de commodities (SEBRAE, 2020). Com isso, este conceito propõe preços mais justos e condições comerciais adequadas aos empreendedores rurais (FAIRTRADE UK, 2020). Porém, somado a esses aspectos, fomenta condições de trabalho éticas e sustentabilidade local (SEBRAE, 2020; FAIRTRADE UK, 2020). Ao prover educação econômica aos produtores de baixa renda, as estratégias que se apoiam no comércio justo possibilitam autonomia, crescimento econômico de seus empreendimentos e condições de vida melhores (FAIRTRADE UK, 2020). Permitem ainda a sustentação econômica de cooperativas e de produtores que produzem em pequena escala (KHMARA; KRONENBERG, 2018).

A fim de garantir confiabilidade, o comércio justo é difundido sob forma de certificado. Os princípios que regem a certificação global podem ser conferidos abaixo (SEBRAE, 2020):

1. Transparência e corresponsabilidade na gestão da cadeia produtiva e comercial.

2. Relação de longo prazo que ofereça treinamento e apoio aos produtores e acesso às informações do mercado.

3. Pagamento de preço justo no recebimento do produto, além de um bônus que deve beneficiar toda a comunidade, e de financiamento da produção ou do plantio, ou a antecipação do pagamento da safra quando necessário.

4. Organização democrática dos produtores em cooperativas ou associações.

5. Respeito à legislação e às normas (por exemplo, trabalhistas) nacionais e internacionais.

6. O ambiente de trabalho deve ser seguro e as crianças devem frequentar a escola.

7. 0 meio ambiente deve ser respeitado.

As empresas que podem utilizar o selo Fair Trade globalmente devem realizar o pagamento dessas licenças por meio de iniciativas locais ou diretamente à instituição Fairtrade Labelling Organizations International (FLO) (SEBRAE, 2020). Apesar do destaque dado aos aspectos sociais, o princípio de preservação do meio ambiente inclui critérios como o "banimento de certos tipos químicos nocivos, redução de pesticidas e promoção de métodos naturais biológicos para preservar o solo e a biodiversidade" (AMBEC, 2014, p. 56). Práticas como o corte rotacional e o cultivo de baixa escala são incluídas nesse sentido (AMBEC, 2014). Tem sido comum, portanto, produtos com o selo fair trade de origem orgânica (BABIER; MARKANDYA, 2013).

Barbier e Markandya (2013) argumentam que as compras de produtos com o certificado de comércio justo fortalecem a economia dos países em desenvolvimento, já que fornecem grande parte das matérias-primas de origem agrícola e pecuária. Além de produtos de origem rural, outros itens, como objetos e vestuários, têm sido manufaturados conforme os princípios do fair trade. Os modelos de negócios que utilizam como proposta de valor o comércio justo inovam sistematicamente quando provocam mudanças tecnológicas e sociais.

\section{Economia distribuída}

Esse conceito propõe que todo o ciclo de vida de um produto e/ou serviço esteja distribuído em unidades próximas, entre as quais as atividades são organizadas de modo descentralizado e em um sistema econômico no qual o usuário também atua na produção. 
Assim, entende-se que unidades pequenas e próximas facilitem a viabilização de produções em pequena escala, bem como a maior ação dos usuários na manufatura. Essa atuação pode ocorrer por meio da cocriação e/ou da personalização de itens produzidos sob demanda ou mesmo alocando atividades manufatureiras para os usuários de produtos e/ou serviços. Existem ainda configurações de economia distribuída nas quais a participação do usuário também acontece na distribuição/comercialização. Em todas as possibilidades acima citadas visa-se a geração e a distribuição de valor e a maior equidade socioeconômica (ULIANA; SANTOS, 2017; VEZZOLI et al., 2020).

O conceito de economia distribuída propõe um sistema conectado e/ou com algumas unidades atuando de maneira independente, mas em rede, visando permitir ainda o compartilhamento de conhecimento ou mesmo de energia excedente (ULIANA; SANTOS, 2017). Esse tipo de configuração, em que todas as fases do ciclo de vida acontecem próximas umas das outras, estimula ao uso dos recursos locais (SANTOS et al., 2019). Vale ressaltar que esses recursos podem vir dos resíduos de outros participantes da rede, ocorrendo sob um modelo similar ao cluster ecológico, porém com foco em dar oportunidade econômica aos pequenos empreendimentos.

Essa proposta visa ainda o fortalecimento dos stakeholders com base na sustentação em rede e por meio da resiliência (SANTOS et al., 2019). A economia distribuída propõe melhor adaptação dos sistemas produtivos em meio a obstáculos econômicos, já que, divido em pequenas unidades próximas, o sistema paralisa por completo quando uma unidade é abalada (VEZZOLI et al., 2020).

Nota-se assim que as estratégias baseadas na economia distribuída podem possibilitar o crescimento econômico pautado na descentralização econômica e na distribuição de renda.

\section{Fundamentações de um design para a economia verde}

A fim de compreender a relação da economia verde com os conceitos derivados dela, os autores propõem a representação visual abaixo (figura 1) ${ }^{19}$. Ao observar as prescrições sob a perspectiva do design, percebe-se que aquelas que apresentam maior ênfase sistêmica estão menos dependentes da atividade industrial, pois inovam com a transformação das relações entre os stakeholders e, assim, somam valor sem necessariamente lançar novos produtos tangíveis. Considera-se, então, mais adequadas à proposta da economia verde, uma vez que viabilizam o crescimento econômico dissociado da exploração ambiental enquanto permitem a promoção de soluções que incluem o âmbito social.

\footnotetext{
19 O tamanho das áreas dos diagramas de cada conceito foi feito de modo a representar visualmente o volume de pesquisas encontradas sobre cada conceito, na RBS. Já a largura e altura dos mesmos seguem a necessidade de expor a abrangência de soluções - se são insulares ou sistêmicas. As intersecções dos diagramas representam justamente as semelhanças em abordagens e estratégias.
} 


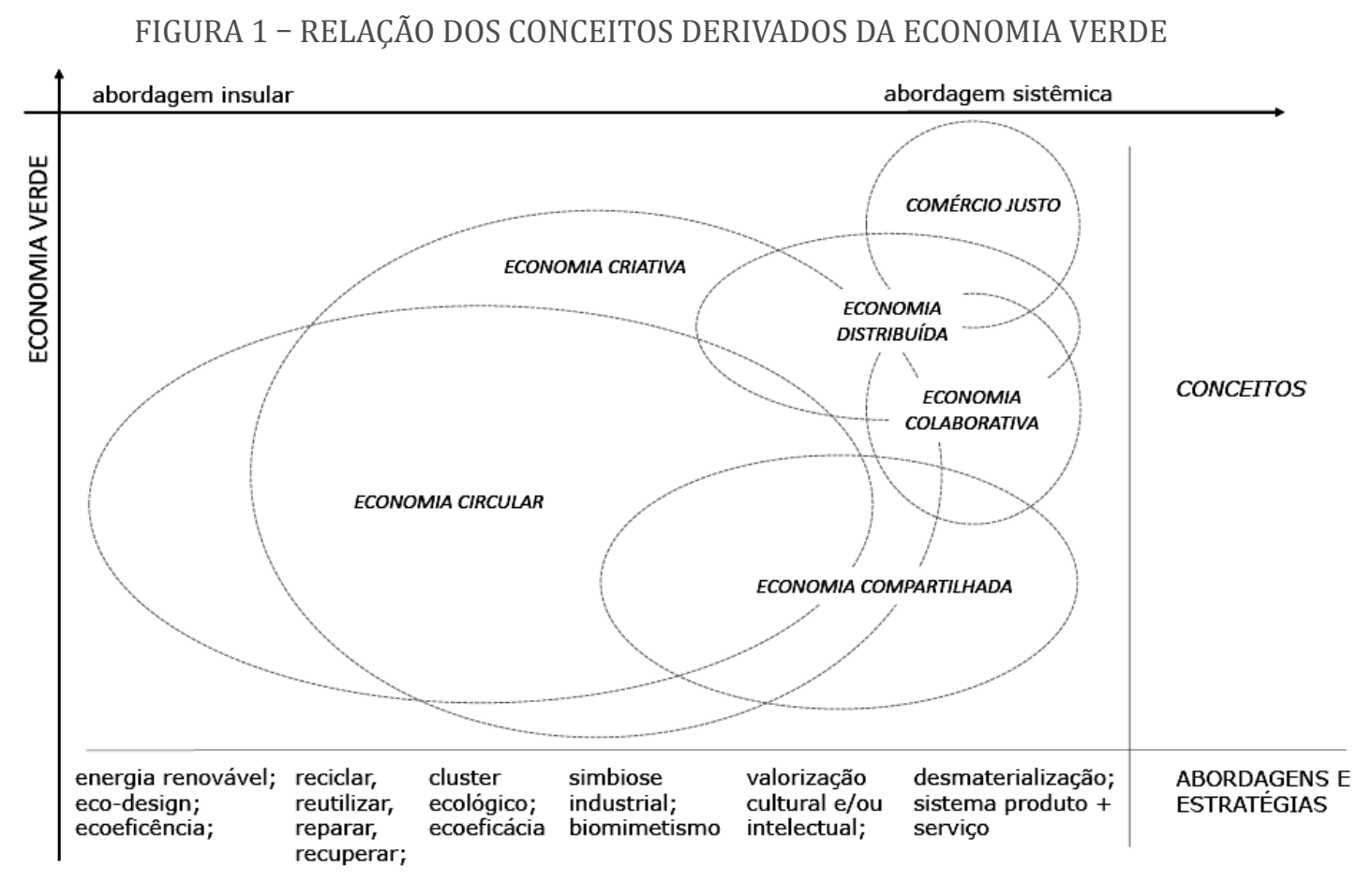

FONTE: A autora (2020).

Partindo dessa observação, destacam-se cinco caminhos de soluções, entre abordagens e estratégias, que fundamentam um design para a economia verde:

1. Desmaterialização: com essa estratégia, procura-se reduzir o uso de materiais, seja do item principal a ser entregue, seja de embalagens. ou mesmo pela exclusão de todo o produto final. Ao se desmaterializar um produto por meio de um serviço que o substitui integral ou parcialmente, a criação volta-se à experiência do consumidor. Com isso, as empresas podem avaliar um custo inicial de menor risco, já diminuindo a dependência da estocagem. No caso da necessidade de estoque/armazenamento e/ou transporte, quando bem planejada, a desmaterialização permite redução do uso do espaço e facilidade logística enquanto reduz a emissão de gases do tipo GEE. Ao utilizar a desmaterialização, a ênfase na economia verde depende da redução de materiais de alto impacto ambiental e da criação de experiências de consumo que eduquem o consumidor para ampliar a visão sobre o uso consciente dos impactos negativos, conforme as suas ações.

2. Manutenção e reparo: essa estratégia demonstra um caminho bastante adequado ao design e à economia verde, já que envolve criação e geração de trabalho. Isso pode valorizar a atividade profissional de artesãos como sapateiros, alfaiates, carpinteiros, bordadeiras, entre diversos outros. Pode-se ainda criar novas perspectivas de trabalho para jovens, dando ênfase em atividades profissionais que envolvem criatividade e trabalho braçal ${ }^{20}$.

\footnotetext{
${ }^{20}$ Vale destacar que o ODS 8 - promover o crescimento econômico sustentado, inclusivo e sustentável, emprego pleno e produtivo e trabalho decente para todos, tem como uma de suas metas a criação de postos de trabalho para os jovens (ONU, 2015).
} 
Se atrelado a isto o uso da tecnologia contribuir na identificação de indivíduos dispostos a trabalhar na manutenção de produtos, a economia verde é intensificada, proporcionando oportunidade e fluxo de trabalho e geração de renda enquanto diminui a obsolescência de um modo geral.

3. Simbiose industrial: os sistemas pautados em stakeholders locais e próximos tendem a criar resiliência econômica pois descentralizam atividades e capitalização. A simbiose industrial é uma estratégia que tem bastante afinidade com o conceito de economia distribuída em si, contudo não envolve o consumidor final. Esse tipo de abordagem permite a participação de artesãos e de micro e pequenas empresas em atividades produtivas, mesmo com uma produtividade considerada baixa em relação a das empresas médias e grandes. Sob a perspectiva da economia verde, isto implica em negociação financeira transparente e que permita poder de barganha a todos os envolvidos - condição esta similar à do comércio justo. Se somada à diversidade intelectual, permite favorecer a participação de empresas voltadas tanto à alta quanto à baixa tecnologia, sejam startups, sejam comunidades de inovação social, por exemplo.

4. Sistemas de produto mais serviço sustentáveis (S.PSS): essa abordagem possibilita a interação entre stakeholders, possuindo assim amplitude sistêmica. Com isso, tem-se valor parcial ou integralmente dissociado da materialidade. Assim, é possível criar um modelo negócio e/ou entregas condizentes com a economia verde, já que é possível promover o crescimento econômico enquanto se beneficia a sociedade e se preserva o meio ambiente.

5. Valorização intelectual e/ou cultural: ao possibilitar que se some valor aos modelos de negócios, sistemas, produtos e/ou serviços a partir do valor simbólico, essa abordagem permite ao design lidar com uma de suas principais competências, a criatividade. Quando atrelada à sustentabilidade econômica, permite fortalecer os valores tradicionais relativos aos processos produtivos, selecionando aqueles que possuem menor pegada ecológica e/ou que fortalecem a autoestima e a autonomia econômica de grupos/comunidades. A valorização intelectual pode ser bastante significante, conforme descrito na economia criativa, e, ainda, direcionada ao fortalecimento da diversidade etária, de identidade de gênero e de etnia para a ocupação de postos de trabalho. É uma abordagem que pode ser amplamente explorada para a economia verde, seja em etapas do processo produtivo, como na escolha de temáticas/conceitos; de materiais e; de métodos produtivos, e seja para permear todo o modelo de negócios.

\section{Considerações finais}

A ONU (2020) e suas agências exerceram um papel fundamental não apenas na legitimação da economia verde, mas no modo esse conceito é empregado na indústria e no mercado global (BOHM et al., 2012; LOISEAU et al., 2016; LEDERER et al., 2018). Nesse sentido, os esforços da ONU (2020) contribuíram para que as nações e grandes organizações percebessem o papel da dimensão econômica da sustentabilidade na preservação e no restauro dos recursos naturais. O Protocolo de Quioto (1998), o GGND (2009), o GEI (2012) e os ODS (ONU, 2015) viabilizaram investimentos em tecnologia e infraestrutura limpa, bem como em práticas que facilitem a equidade social, seja por meio de subsídios diretos e/ou pela possibilidade da capitalização de ações empresariais para a economia verde. 
Esses aspectos demonstram que o crescimento econômico tem sido o catalisador da economia verde (WORLD BANK, 2012; BÖHM et al. 2012; PHALEN, 2020). Assim, fica anunciada uma mudança no papel da economia, retirando-a da posição de problema para solução na busca pelo desenvolvimento sustentável.

No entanto, sob o domínio de contextos cuja lógica do livre mercado prevalece, corre-se o risco de a economia verde ser utilizada como um meio para se acumular capital e manter a hegemonia econômica dos países desenvolvidos e das grandes empresas. Isto porque a ênfase na eficiência do uso de recursos naturais e na exploração da força de trabalho já acontece no sistema econômico predominante e insustentável. Logo, é necessário se aprofundar em soluções focadas nas externalidades, propiciando a regeneração ambiental e a equidade socioeconômica, e não apenas em mais capitalização.

Os conceitos acima investigados, embora de fato envolvam design e fundamentem a economia verde original, também podem recair nas mesmas falhas de projeção das externalidades ambientais e/ou sociais. Observa-se, por exemplo, que a economia circular tem ganhado destaque em diferentes áreas e setores, inclusive no design e na moda. Isso tem ocorrido por meio de pesquisas e de incentivos financeiros derivados do GGND (2009) e devem aumentar com a proposta da nova Bauhaus (NEW EUROPEAN BAUHAUS, 2021). Esse propósito estimula as atividades criativas enquanto gera trabalho e renda. Entretanto, se não houver esforços para mudar a frequência de lançamento de novos produtos, não se sabe se a economia circular viabilizará de fato uma transição para a sustentabilidade ou apenas será utilizada como justificativa para dar continuidade à aceleração industrial.

As inovações em economia compartilhada são outro exemplo de fácil risco de falha no que diz respeito à economia verde. A informalização pode oportunizar a rápida recolocação de profissionais que estão desempregados. No entanto, pode, paradoxalmente, propiciar a ampliação de postos de trabalho com condições precárias e ainda facilitar a centralização do lucro, favorecendo a desigualdade socioeconômica. Modelos de negócios de economia compartilhada voltados ao transporte de passageiros, por exemplo, também provocar impactos negativos no meio ambiente. Isso ocorre quando fomentam a diminuição do uso otimizado de ônibus públicos e, assim, dão chance à maior emissão de GEE.

Outro aspecto a ser considerado ao se visar o fomento em uma transição para a sustentabilidade por meio de um design para a economia verde é o enfoque dado na macro ou na microeconomia. Uma vez observado que os estudos e os avanços da economia verde têm sido direcionados à construção de políticas que visam a regulamentação do comércio internacional e a sua adesão por grandes empresas, é no aspecto microeconômico que ela deve ser intensificada ao se visar construir um novo paradigma econômico, mais equânime.

Por isso, os autores entendem que um design orientado ao conceito integral e original da economia verde se faz necessário, já que não se trata de usar fragmentos de soluções e sim uma abordagem sistêmica. Entretanto, é apenas um começo. Somado a isso, sugere-se que futuros trabalhos se pautem na identificação de princípios que guiem profissionais criativos a um olhar analítico sobre as externalidades. É o que os autores pretendem realizar tendo em vista a busca pela aplicabilidade do conceito pelo design. Simultaneamente, este artigo fica como um convite para que outros investigadores interessados em design e moda colaborem com uma transição para a sustentabilidade, enquanto se amplia a percepção de como criar valor e oportunizar prosperidade individual e coletiva preservando o planeta. 


\section{Referências}

ALTENBURG, Tilman; Rodrick, Dani. Green industrial policy: accelerating structural change towards wealthy green economies. In: ALTENBURG, Tilman; ASSMANN, Claudia. Green industrial policy. Concept, policies, country experiences. Geneva; Bonn: UN Environment; German Development Institute / Deutsches Institut für Entwicklungspolitk (DIE), 2017, p. 2-9.

AMBEC, Stefan. Gaining competitive advantage with green policy. In: Green industrial policy. Concept, Policies, Country Experiences. In ALTENBURG, Tilman; ASSMANN, Claudia. Green industrial policy. Concept, policies, country experiences. Geneva; Bonn: UN Environment; German Development Institute / Deutsches Institutfür Entwicklungspolitk (DIE), 20177, p. 39-43.

BAKKER, Conny; WANG, Feng; JACO, Huisman; DEN HOLLANDER, Marcel. Products that go round: exploring product life extension through design. Journal of Cleaner Production, $\mathrm{V}$. 69, p. 10-16, 2014. Disponível em: http://dx.doi.org/10.1016/j.jclepro.2014.01.028. Acesso em: 20 nov. 2020.

BARBIER, Edward; MARKANDYA, Anil. A new blueprint for a green economy. Abingdon UK: Routledge, 2014.

BARNES, Stuart J.; MATTSSON, Jan. Understanding current and future issues in collaborative consumption: a four-stage Delphi study. Technological Forecasting and Social Change, v. 114 , p. 200-211, 2016.

BÖHM, Steffen.; MISOCZKY, Maria Ceci. e MOOG, Sandra. Greening capitalism? A marxist critique of carbon markets. Organization Studies, n. 33 v. 11, p. 1617-1638, 2012.

BRAUGART, Michael; McDONOUGHT, William; BOLLINGER, Andrew. Cradle-to-cradle design: creating healthy emissions e a strategy for eco-effective product and system design. Journal of Cleaner Production, v. 1, n. 12, p. 1-12, 2006.

BRUNDTLAND, Gro Harlem. Report of the world commission on environment and development "our common future". Journal of International Development, v. 1, n. 2, p. 284-287, 1989. Disponível em: https://sustainabledevelopment.un.org/content/ documents/5987our-common-future.pdf. Acesso em: 10 nov. 2020.

CATO, Molly Scott. Green economics: Putting the planet and politics back into economics. Cambridge Journal of Economics, v. 36, n. 5, p. 1033-1049, 2012. 
CASTRO, Francisco Gómez; FIGUEIREDO, Luiz Fernando. A economia criativa como proposta de valor nos modelos de negócios. Navus - Revista de Gestão e Tecnologia, v.6(3) p.111$122,2016$.

CESCHIN, Fabrizio. The introduction and scaling up of sustainable product-service systems. 2012. 374 f. Tese (Doutorado em Design) - Departamento de Design Industrial, Arte, Comunicação e Moda (INDACO), Politécnico de Milão, Milão, 2012.

CESCHIN, Fabrizio; GAZIULUSOY, Idil. Evolution of design for sustainability: From product design to design for system innovations and transitions. Design Studies, v. 47, p. 118-163, 2016. Disponível em: http://dx.doi.org/10.1016/j.destud.2016.09.002. Acesso em: 26 nov. 2020.

CORPORATE KNIGHTS. 2020 Global 100 ranking. Disponível em: https://www. corporateknights.com/reports/2020-global-100/2020-global-100-ranking-15795648/. Acesso em: 26 nov. 2020.

DANTAS, Denise; BERTOLDI, Cristiane; TARALLI, Cibele H. Materiais e criação em design e arquitetura: compartilhando experiências para a economia criativa. Revista do Programa de Pós-Graduação em Arquitetura e Urbanismo da FAUUSP, v. 24-42, p. 110, 2017. Disponível em: https://doi.org/10.11606/issn.2317-2762.v24i42p110-126. Acesso em: 10 nov. 2020.

DATA SEBRAE. Informações sobre a sustentabilidade nos pequenos negócios. Disponível em: https://datasebrae.com.br/sustentabilidade/. Acesso em: 10 nov. 2020.

DE LOS RIOS, Irel Carolina; CHARNLEY, Fiona J. S. Skills and capabilities for a sustainable and circular economy: The changing role of design. Journal of Cleaner Production, v. 160, p. 109-122, 2017.

DEN HOLLANDER, Marcel C. den.; BAKKER, Conny A.; HULTINK, Erik J. Product Design in a Circular Economy: Development of a Typology of Key Concepts and Terms. Journal of Industrial Ecology, v. 21-23, p. 517-525, 2017.

DE RIVERA, Javier de; GORDO, Ángel; CASSUDY, Paul.; APESTEGUÍA, Amaya. A netnographic study of P2P collaborative consumption platforms' user interface and design. Environmental Innovation and Societal, v. 23, p. 11-27, 2017.

DESPEISSE, Melaine; BAUMERS, Martin; BROWN, Phil et al. Unlocking value for a circular economy through 3D printing: a research agenda. Technological Forecasting and Social Change, v. 115, p. 75-84, 2017. 
DUARTE, Gabriela Garcez (2020). Design para a economia Verde: aprendizagem reflexiva em organizações. Documento de qualificação (Doutorado em Design) - Setor de Ciências Humanas, Universidade Federal do Paraná, Curitiba, 2020.

ELLENMACARTHURFOUNDATION,Disponívelem:https://www.ellenmacarthurfoundation. org/. Acesso em: 3 nov. 2020.

FAIR TRADE. Disponível em: https://www.fairtrade.org.uk/. Acesso em: 7 nov. 2020.

GAN, Mi; YANG, Shuai; LI, Dandan; WANG, Mingfei; CHEN, Si; XIE, Ronghui; LIU, Jiyang. A novel intensive distribution logistics network design and profit allocation problem considering sharing economy. Complexity, 2018. Disponível em: https://www.hindawi.com/journals/ complexity/2018/4678358/. Acesso em: 7 nov. 2020.

GAZIULUSOY, Idil. Systems innovations for business sustainability. Entrevista realizada em 10 set.2020, Nova Zelândia. Informação verbal.

GEELS, Frank W.; SCHOT, Johan. Typology of sociotechnical transition pathways. Reseacrh Policy, v. 36, p. 399-417, 2007.

GEELS, Frank W. Ontologies, socio-technical transitions (to sustainability), and the multilevel perspective. Research Policy, v. 39-44, p. 495-510, 2010.

GEENHUIZEN, Marina van; QING, Ye. Responsible innovators: open networks on the way to sustainability transitions. Technological Forecasting and Social Change, v. 87, p. 28-40, 2014.

GLOBAL GREEN NEW DEAL: An Update for the G20 Pittsburgh Summit, 2009. Disponível em: https://wedocs.unep.org/bitstream/handle/20.500.11822/7736/-Global\%20 Green\%20New\%20Deal_\%20An\%20Update\%20for\%20the\%20G20\%20Pittsburgh\%20 Summit-2009880.pdf?sequence=3\&isAllowed=y. Acesso em: 7 nov. 2020.

HARVEY, DAVID. 17 contradições e o fim do capitalismo. Trad. Rogério Bettoni. São Paulo: Boitempo, 2016.

HENDERSON, Hazel. Growing the green economy globally. International Journal of Green Economics, v. 1, n. 3-4, p. 276, 2007. Disponível em: http://www.inderscience.com/link. php?id=13060. Acesso em: 3 nov. 2020.

ITAMARATY. Disponível em: http://www.itamaraty.gov.br/pt-BR/politica-externa/diplomaciaeconomica-comercial-e-financeira/15586-brasil-g20. Acesso em: 13 nov. 2020. 
JANKOWSKI, Wanda. The value of design - factfind repport. Design Council, 2007. Disponível em: https://www.designcouncil.org.uk/sites/default/files/asset/document/ TheValueOfDesignFactfinder_Design_Council.pdf. Acesso em: 7 de nov. 2020.

KHMARA, Yaryna; KRONENBERG, Jackub. Degrowth in business: an oxymoron or a viable business model for sustainability? Journal of Cleaner Production, v. 177, p. 721-731, 2018.

KULP, Heather Scheiwe; KOOL, Amanda L. You help me, he helps you: dispute systems design in the sharing economy. Washington University Journal of Law \& Policy, v. 48, p. 179-230, 2015. Disponível em: http://openscholarship.wustl.edu/cgi/viewcontent. cgi?article=1881\&context=law_journal_law_policy. Acesso em: 7 nov. 2020.

KYOTO PROTOCOL, 1998. Disponível em: https://unfccc.int/resource/docs/convkp/kpeng. pdf. Acesso em: 10 nov. 2020.

LIGHT, Ann; MISKELLY, Clodagh. Sharing economy vs sharing cultures? Designing for social, economic and environmental good. Interaction Design and Architecture(s), v. 24-31, p. 49-62, 2015.

LOISEAU, Eleonore.; SAIKKU, Laura.; ANTIKAINEN, Riina. et al. Green economy and related concepts: an overview. Journal of Cleaner Production, v. 139, p. 361-371, 2016.

MANZINI, Ezio; VEZZOLI, Carlo. Desenvolvimento de produtos sustentáveis - os requisitos ambientais dos produtos industriais. São Paulo: Editora EDUSP, 2002.

MARTIN, Chris J. The sharing economy: A pathway to sustainability or a nightmarish form of neoliberal capitalism? Ecological Economics, v. 121, p. 149-159, 2016.

MEADOWS, Donella H.; MEADOWS, Dennis L.; REANDERS, Jorgen; NEHRENS, William W. The limits to growth, 1972. Disponível em: https://donellameadows.org/wp-content/ userfiles/Limits-to-Growth-digital-scan-version.pdf . Acessado em: 7 nov. 2020.

MENDOZA, Joan M. F.; SHARMINA, Maria; GALLEGO-SCHMID, Alejandro; HEYES, Graeme.e AZAPAGIC, Adisa. Integrating backcasting and eco-design for the circular economy: the BECE framework. Journal of Industria Ecology, v. 21-23, p. 526-544, 2017.

MINISTÉRIO DO MEIO AMBIENTE. Protocolo de Quioto. Disponível em: https://www.mma.gov. br/clima/convencao-das-nacoes-unidas/protocolo-de-quioto.html. Acesso em: 24 nov. 2020.

NAÇÕES UNIDAS. Disponível em: https://nacoesunidas.org/acao/meio-ambiente/. Acesso em: 22 nov. 2020. 
ONU NEWS. Disponível em: https://news.un.org/pt/story/2020/07/1719331. Acesso em: 7 nov. 2020.

OXFAM. Bilionários do mundo tem mais riqueza que $\mathbf{6 0 \%}$ da população. Disponível em: https://www.oxfam.org.br/noticias/bilionarios-do-mundo-tem-mais-riqueza-do-que60-da-populacao-mundial/. Acesso em: 10 nov. 2020.

OXFAM. Crise pra quem? Grandes corporações lucram bilhões durante a pandemia. Disponível em: https://www.oxfam.org.br/blog/crise-para-quem-grandes-corporacoeslucraram-bilhoes-durante-a-pandemia/. Acesso em: 10 Novembro 2020.

PEARCE, David; MARKANDYA, Anil; BARBIER, Edward. The blueprint for a green economy, 1989. Disponível em: https://www.researchgate.net/publication/39015804_Blueprint_ for_a_Green_Economy. Acesso em: 10 nov. 2020.

PEARCE David; MORAN, Dominic. The economic value of biodiversity Iunch — the World Conservation Union. 1 ed. Londres: Earthscan Publications Limited, 1994

PETROPOULOS, Georgis. Collaborative economy: market design and basic regulatory principles. Intereconomics, v. 52-56, p. 340-345, 2017. Disponível em: http://link.springer. com/10.1007/s10272-017-0701-8. Acesso em: 7 nov. 2020.

PHALEN, Christopher. Investing to achieve the UN Sustainable Development Goals, 2020. Disponível em: https://www.ussif.org/files/Publications/USSIF_Investing\%20to\%20 Achieve\%20the\%20UN\%20SDGs_FINAL.pdf. Acesso em: 10 nov. 2020.

PORTAL DE PERIÓDICOS CAPES - MEC. Disponível em: https://www-periodicos-capesgov-br.ezl.periodicos.capes.gov.br. Acesso em: 20 nov. 2019.

REPORTE OF UNITED NATIONS CONFERENCE OF THE HUMAN ENVIRONMENT, 1973. PDF. Disponível em: https://www.un.org/ga/search/view_doc.asp?symbol=A/CONF.48/14/REV.1 Acesso em: 10 nov. 2020.

RIVERA, Javier; GORDO, Ángel; CASSIDY, Paul; APESTEGUÍA, Amaya. A netnographic study of P2P collaborative consumption platforms' user interface and design. Environmental, Innovation and Societal Transitions, v. 23, p. 11-27, 2018. Disponível em: https:// linkinghub.elsevier.com/retrieve/pii/S2210422416300880. Acesso em: 7 jul. 2020. https:// doi.org/ 10.1016/j.eist.2016.09.003.

SANDRONI, PAULO (org). Novíssimo dicionário de economia. São Paulo: Editora Best Seller, 1999. 
SANTOS, Aguinaldo dos (org.) Design para a sustentabilidade: dimensão econômica. $1^{\text {a }}$ ed. Curitiba: Insight Editora, 2019.

SCHUMACHER, Emst F. 0 negócio é ser pequeno: um estudo de economia que leva em conta as pessoas. 4. ed. São Paulo: Zahar Editores, 1973.

SCHEEL, Carlos. Beyond sustainability: transforming industrial zero-valued residues into increasing economic returns. Journal of Cleaner Production, v. 131, p. 376-386, 2016.

SEBRAE. Como o SEBRAE atua na economia criativa. Disponível em: https://www. sebrae.com.br/sites/PortalSebrae/segmentos/economia_criativa/como-o-sebrae-atuano-segmento-de-economia-criativa,47e0523726a3c510VgnVCM1000004c00210aRCRD. Acesso em: 7 nov. 2020.

SEBRAE. Comunidades incorporam design à moda. Disponível em: https://blog.sebrae-sc. com.br/parceria-leva-design-de-moda-a-comunidades-carentes/. Acesso em: 7 nov. 2020.

SEBRAE. 0 que é Fair Trade (comércio justo). Disponível em: https://www.sebrae.com. br/sites/PortalSebrae/artigos/o-que-e-fair-trade-comercio-justo,82d8d1eb00ad2410Vgn VCM100000b272010aRCRD. Acesso em: 7 nov. 2020.

SEBRAE. Entenda as diferenças entre microempresa, pequena empresa e MEI. Disponível em: https://www.sebrae.com.br/sites/PortalSebrae/artigos/entenda-as-diferencas-entremicroempresa-pequena-empresa-e-mei,03f5438af1c92410VgnVCM100000b272010aRC RD. Acesso em: 8 nov. 2020.

SHU-HSIANG, Chen; JAITIN, Nosaongkhla; DONALDSON J. Ana. From vision to action - A strategic planning process model for open educational resources. Procedia - Social and Behavioral Sciences, v.174, p. 3707-3714, 2015.

ULIANA, Iana Perez; SANTOS, Aguinaldo dos. Distributed economies through open design and digital manufacturing, v.3 n.4, p. 21-28, 2017.

UNITED NATIONS ENVIRONMENT PROGRAM (UNEP). Disponível em: https://www. unenvironment.org. Acesso em: 25 nov. 2019.

UNITED NATIONS ENVIRONMENT PROGRAM (UNEP) e SOCIETY OF ENVIRONEMNTAL TOXICOLOGY AND CHESMISTRY (SETAC), 2012. Greening the economy through life cycle thinking. Disponível em: https://www.lifecycleinitiative.org/wp-content/ uploads/2013/03/2012_LCI_10_years_28.3.13.pdf. Acesso em: 1 maio 2021. 
UNITED IN SCIENCE, 2019: PDF. Disponível em: https://wedocs.unep.org/bitstream/ handle/20.500.11822/30023/climsci.pdf. Acesso em: 25 nov. 2019.

VASQUES, Rosana Aparecida; KORIA, Mikko; SANTOS, Maria Cecília Loschiavo dos. Why do I love you Vaatelainaamo? Analysis of motivations, barriers and opportunities in a finnish service for sharing clothes. The Design Journal, v. 20, p. 721-731, 2017.

VERBOVEN, Hans; VANHERCK, Lise. The sustainability paradox of the sharing economy. UmweltWirtschaftsForum, v. 24-34, p. 303-314, 2016. Disponível em: http://link.springer. com/10.1007/s00550-016-0410-y. Acesso em: 7 nov. 2020.

VEZZOLI, Carlo; KOHTALA, Cindy; AMRIT, Srinivasan et al. Sistema produto + serviço: fundamentos. Trad. Aguinaldo dos Santos. Curitiba: Insight Editora, 2018

VEZZOLI, Carlo.;GARCIA, Brenda; KOHTALA, Cindy (Eds.). Designing sustainability for all the design of sustainable product-service system applied to distributed economies, 2021. Disponível em: https://www.springer.com/it/book/9783030662998. Acesso em: 7 maio 2021.

WORLD BANK. Inclusive green growth: the pathway to sustainable development, v. 6B, p.171, 2012. 